\title{
Articles
}

\section{Analysis of the Wettability of Partially Fluorinated Polymers Reveals the Surprisingly Strong Acid-Base Character of Poly(vinylidene Fluoride)}

\author{
Sangwha Lee, ${ }^{*}$ Joon-Seo Park, ${ }^{\dagger}$ and T. Randall Lee ${ }^{\ddagger}, *$ \\ Department of Chemical and Bio Engineering, Kyungwon University, San 65 Bokjeong-Dong, Sujeong-Ku, Seongnam, \\ Gyeonggi-Do 461-701, Korea.*E-mail: lswha@kyungwon.ac.kr \\ $\dagger$ Department of Chemistry, Eastern University, 1300 Eagle Road, St. Davids, PA 19087-3696, USA \\ ${ }^{\ddagger}$ Department of Chemistry, University of Houston, 4800 Calhoun Road, Houston, TX 77204-5003, USA \\ *E-mail: trlee@uh.edu \\ Received March 23, 2010, Accepted August 25, 2010
}

\begin{abstract}
The wettabilities of the partially fluorinated polymers (ethylene-tetrafluoroethylene copolymer (ETFE), ethylenechlorotrifluoroethylene copolymer (ECTFE), and poly(vinylidene fluoride) (PVDF)) were investigated by contact angle measurements. Zisman plots for ETFE and ECTFE exhibited linear relationships, while the Zisman plot for PVDF showed a slight curvature, which was interpreted to indicate strong non-dispersive interactions between the surface and the contacting liquids. The Lifshitz-van der Waals forces of the fluoropolymers were estimated to increase in the order of ETFE $<$ PVDF $<<$ ECTFE. An evaluation of the polar or "acid-base" interaction energies showed that PVDF, which possesses the most acidic hydrogens among the examined fluoropolymers, has the strongest acid-base interactions.
\end{abstract}

Key Words: Poly(vinylidene fluoride), Wettability, Surface dipoles, Acid-base interactions, Dipole-dipole interactions

\section{Introduction}

Fully fluorinated polymers, including poly(tetrafluoroethylene) (PTFE), fluoroethylene-propylene copolymer (FEP), and polyperfluoroalkoxyethylene (PFA), are nonpolar materials consisting of strong $\mathrm{C}-\mathrm{F}$ and $\mathrm{C}-\mathrm{C}$ inter-atomic bonds that give rise to their robust chemical structures and superior interfacial properties. ${ }^{1,2}$ Partially fluorinated polymers, including ethylenetetrafluoroethylene copolymer (ETFE), ethylene-chlorotrifluoroethylene copolymer (ECTFE), and poly(vinylidene fluoride) (PVDF), in which selected fluorine atoms in fully fluorinated polymers are replaced by foreign atoms (e.g., hydrogen or chlorine as illustrated in Figure 1), exhibit increased polarity because the electronegativities of the substituting elements are less than that of fluorine. The spatially symmetrical disposition of the hydrogen (or chlorine) and fluorine atoms along the polymer chain gives rise to the polarity, which can influence the solubility, chemical/structural stability, and interfacial properties of the polymers. Furthermore, the wettabilities of partially fluorinated surfaces increase with increasing degree of substitution and proximate distance between $\mathrm{CF}_{2}$ and $\mathrm{CH}_{2}$ units. ${ }^{3,4}$

In contrast to nonpolar fully fluorinated polymers, partially fluorinated polymers can be dissolved in polar solvents, such as organic esters and amines, and readily melt-processed by the standard method of molding and extrusion. ${ }^{5}$ In particular, PVDF, with its high polarity, also possesses outstanding thermal, chemical, and mechanical stabilities, which has led to its widespread use in various applications, such as hybrid nanocomposite membranes, ${ }^{6}$ electrochemically stable electrolytes for lithium batteries, ${ }^{7-9}$ fuel cell membranes based on Nafion-
PVDF blends, ${ }^{10-12}$ and piezoelectric sensor materials utilizing its ferroelectric and pyroelectric properties. ${ }^{13-15}$ Although PVDFbased materials serve as highly stable membranes, polymer electrolytes, and even sensor materials, the characteristic surface wettability of PVDF must be adequately understood to design next-generation PVDF-based materials and devices with enhanced performance.

Ellison and Zisman interpreted the wettability of fluorinated surfaces in terms of the electronegativity of the fluorine atoms and the molecular structure of the probe liquids. ${ }^{16}$ Lee and coworkers extended Zisman's interpretation and showed that the wettability of a surface can be strongly influenced by the distribution of acidic groups and/or surface dipoles across the interface. ${ }^{17-19}$ In the thermodynamic polymer blending and sorption process, it is widely accepted that the $\mathrm{CF}_{2}$ dipoles in PVDF are largely responsible for the intermolecular dipolar interaction. ${ }^{20-25}$ For the technologically important piezo and pyroelectric properties of PVDF, the polymer must be uniaxially oriented to induce a crystalline $\beta$-phase such that $\mathrm{C}$-F dipoles are oriented to form a polar unit cell. ${ }^{20-25}$

With strong surface dipoles caused by alternating $\mathrm{CH}_{2}$ and $\mathrm{CF}_{2}$ units, $\mathrm{PVDF}$ is expected to possess acidic hydrogens because the fluorine atoms highly polarize the neighboring $\mathrm{CH}_{2}$ groups. ${ }^{19}$ In this report, we systematically examine the complex operation of dipole-dipole and acid-base interactions on the surface wettability of partially fluorinated polymers with various contacting liquids. In particular, the non-dispersive interactions arising from surface dipoles and acidity in PVDF will be compared with those in ETFE and ECTFE, which are less polar and possess less acidic hydrogens than PVDF. ${ }^{19}$ 


\section{Materials and Methods}

Materials. The contacting liquids were of the highest purity available from commercial suppliers. Fluoropolymers were obtained from Dupont and Ausimont and can be broadly classified into two major categories: fully fluorinated polymers (Teflon ${ }^{\mathrm{R}}$ PFA and FEP) and partially fluorinated polymers (Tefzel $^{(R)}$ ETFE, Solef ${ }^{R}$ PVDF, and Halar ${ }^{R}$ ECTFE). The molecular structures of the partially fluorinated polymers are shown in Figure 1.

X-ray photoelectron spectroscopy (XPS). X-ray photoelectron spectra of fluoropolymer samples were obtained using a PHI 5700 X-ray photoelectron spectrometer equipped with a monochromic Al K $\alpha$ X-ray source $(\mathrm{h} v=1486.7 \mathrm{eV})$ incident at $90^{\circ}$ relative to the axis of a hemispherical energy analyzer. The spectrometer was operated at high resolution, with a pass energy of $23.5 \mathrm{eV}$, a photoelectron take off angle of $45^{\circ}$ from the surface, and an analyzer spot diameter of $1.1 \mathrm{~mm}$. XPS spectra were collected at room temperature and a base pressure of $2 \times$ $10^{-9}$ Torr for $\mathrm{C} 1 \mathrm{~s}, \mathrm{~F} 1 \mathrm{~s}, \mathrm{O} 1 \mathrm{~s}$, and $\mathrm{Cl} 2 \mathrm{p}$. All binding energies were referenced to the $\mathrm{F} 1 \mathrm{~s}$ at $688.65 \mathrm{eV}$ to eliminate charging effect. The intensities of the peaks were determined with standard curve-fitting software (Multipak V5.0A; Physical Electronics, Inc.) using Shirley background subtraction and Gaussian-Lorentzian profiles.

Contact angle measurements. Advancing and receding contact angles were measured under ambient conditions using a ramé-hart model 100 contact angle goniometer. The contacting liquids were dispensed and withdrawn at the slowest possible speed ( $c a .1 \mu \mathrm{L} / \mathrm{s})$ using a Matrix Technologies Micro-Electrapette 25 . The measurements were performed while maintaining contact between the pipet tip and the drop.

AFM measurements. The surface morphology of pristine polymer samples was measured by Atomic Force Microscopy (AFM) using a PSIA XE-150 in noncontact mode at a scan rate of $1 \mathrm{~Hz}$. The probing tip scanned an area of $10 \times 10 \mu \mathrm{m}^{2}$ at different locations on the sample, followed by post-acquisition processing to give a three-dimensional topographic image and the vertical roughness of the sample surface. The surface roughness was determined from the AFM images by calculating the root-mean-square roughness, $\mathrm{R}_{\mathrm{q}}$, from the standard definition using the XEP data acquisition program.

\section{Results and Discussion}

Characteristics of surface dipoles. As shown in Figure 1, ETFE is an alternating copolymer of ethylene and tetrafluoroethylene units, and ECTFE is an alternating copolymer of ethylene and chlorotrifluoroethylene units. PVDF, which is isomeric with the homopolymer of ETFE, is an addition polymer of 1,1difluoroethene $\left(\mathrm{CH}_{2}=\mathrm{CF}_{2}\right)$, which can be readily polymerized by free-radical initiators to form a semicrystalline polymer. ${ }^{26}$ The alteration of $-\mathrm{CH}_{2}$ - and $-\mathrm{CF}_{2}(\mathrm{or} \mathrm{Cl}, \mathrm{H})$-, that is, an electronpoor group and an electron-rich group gives rise to a distribution of surface dipoles along the backbones of partially fluorinated polymers. ${ }^{19}$ The magnitude of the surface dipoles changes if the distance between the two alternating units is varied. For example, the surface dipoles in ETFE are more widely distri-

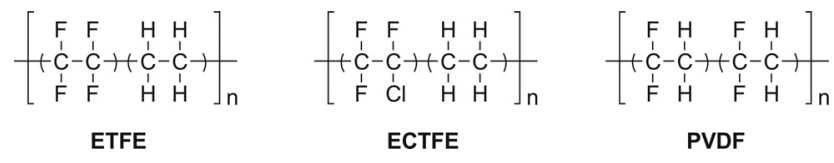

Figure 1. Chemical structures of the partially fluorinated polymers investigated.

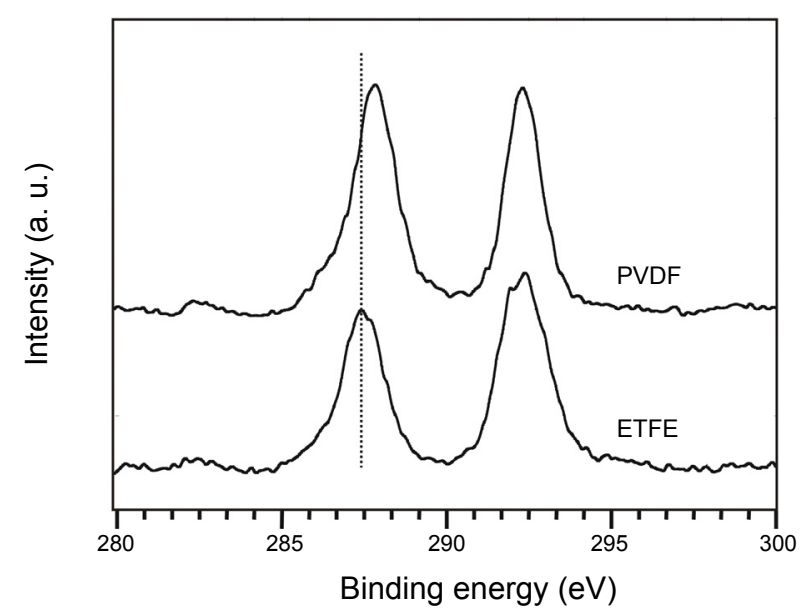

Figure 2. XPS spectra of the $\mathrm{C}_{1 \mathrm{~s}}$ region for ETFE and PVDF.

buted (i.e., further apart), leading to a relatively low density of dipoles when compared to PVDF. The surface dipoles in ECTFE might exhibit multiplicity due to the substitution of $\mathrm{CFCl}$ for $\mathrm{CF}_{2}$, leading to a higher density of dipoles than ETFE and thus enhanced wettability. ${ }^{16,19,27,28}$ Therefore, the magnitude of the surface dipoles of the following partially fluorinated polymers is expected to increase in this order: ETFE $<$ ECTFE $<<$ PVDF.

The fluorine atoms have a strong electron-withdrawing ability, leaving the attached carbon in an electron-deficient state. The electron-withdrawing effect of fluorine decreases with increasing distance along the chain; consequently, the "ionic character" of the hydrogen atom in a $\mathrm{CH}_{2}$ group reasonably decreases upon moving away from $\mathrm{CF}_{2}{ }^{28}$ When one considers the relation of position and proximity of fluorine to hydrogen, the hydrogens of PVDF are the most acidic because fluorines are attached to both neighboring carbons. On the other hand, the hydrogens of ECTFE are the least acidic because the attached chlorine is less electronegative than fluorine. Given these considerations, the acidity of the hydrogen atoms in the partially fluorinated polymers is expected to increase in this order: ECTFE $<$ ETFE $<<$ PVDF. Therefore, we can reasonably predict that PVDF would exhibit the strongest acid-base interaction with probe liquids and ECTFE would exhibit the weakest acidbase interaction among the examined partially fluorinated polymers.

The XPS spectra in Figure 2 of ETFE and PVDF show two main peaks corresponding to the $\mathrm{CF}_{2}$ and $\mathrm{CH}_{2}$ groups, and there is negligible oxygen content. While the $\mathrm{C}_{1 \mathrm{~s}}$ peak for the $\mathrm{CF}_{2}$ groups of both polymers appears at $292 \mathrm{eV}$, the $\mathrm{C}_{1 \mathrm{~s}}$ peak of the $\mathrm{CH}_{2}$ groups in PVDF is shifted by about $1.0 \mathrm{eV}$ toward higher binding energy compared to that of ETFE. The difference is probably due to the enhanced polarization of the $\mathrm{C}-\mathrm{C}$ bonds by the two neighboring electron-withdrawing $\mathrm{CF}_{2}$ groups in PVDF, 
(a)

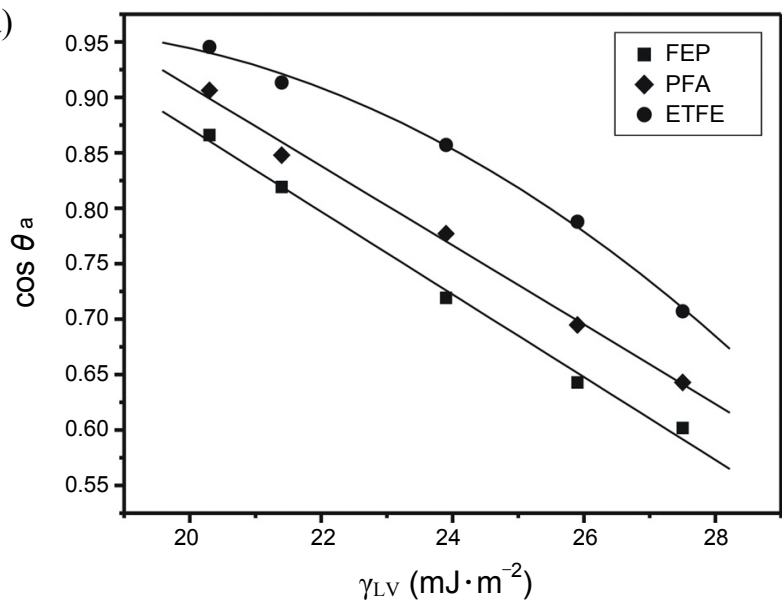

(b)

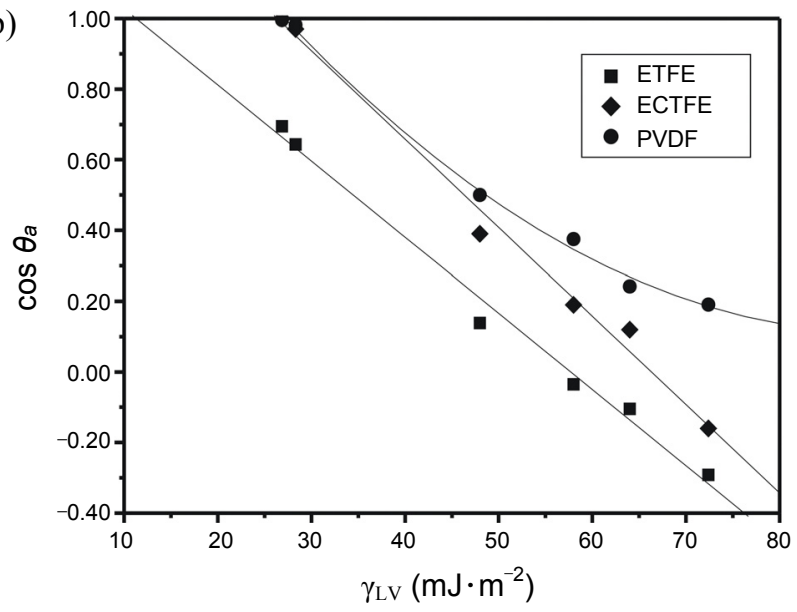

Figure 3. Zisman plots of $\cos \theta_{a} v s . \gamma_{\mathrm{LV}}$ for various probe liquids on selected fluorinated polymers. (a) Nonpolar aprotic liquids: heptane, octane, decane, tridecane, and hexadecane on FEP, PFA, and ETFE. (b) Polar protic liquids: water, formamide, glycerol, ethylene glycol, decanol, and heptanol on ETFE, ECTFE, and PVDF.

which is again consistent with the existence of strong surface dipoles and an enhanced acidity of hydrogens in PVDF when compared to ETFE.

Analysis of Zisman plots. A Zisman plot of $\cos \theta v s . \gamma_{\mathrm{LV}}$ normally gives an approximately straight line that can be extrapolated to the critical surface tension of the solid surface at cos $\theta=1$, unless the contacting liquids form any non-ideal interaction on the solid surface. ${ }^{29}$ Even when $\cos \theta$ is plotted against $\gamma_{\mathrm{LV}}$ of nonhomologous liquids, the graphical points often fall close to a straight line (or in a narrow rectangular band) ${ }^{30}$ We measured the advancing contact angles for several probe liquids in contact with the fluorinated surfaces. As shown in Figure 3a, a homologous series of normal alkane contacting liquids gave a linear Zisman plot for the fully fluorinated FEP and PFA, but a slightly convex plot for the partially fluorinated ETFE.

We chose to use polar protic liquids to gain further insight into the forces operating on the partially fluorinated surfaces. As shown in Figure 3b, a collection of polar protic contacting liquids gave an approximately linear Zisman plot for ETFE and ECTFE, but a concave plot for PVDF, which indicates strong non-dispersive interactions (dipole-dipole and/or acid-base

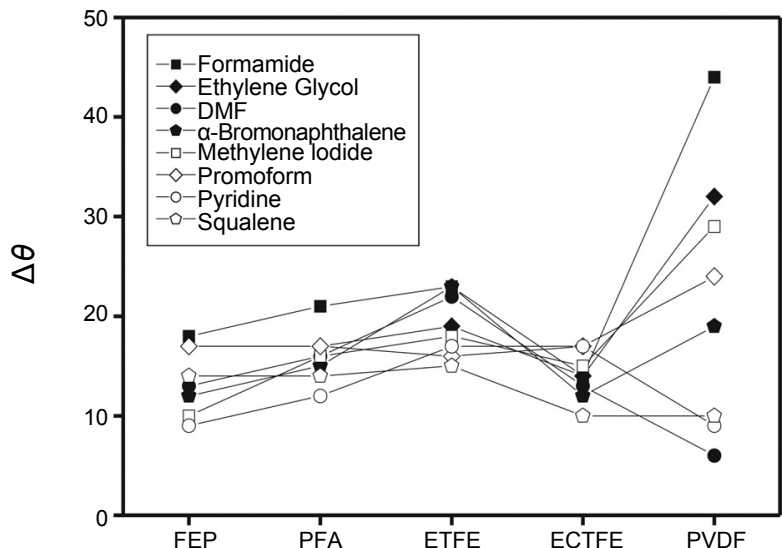

Figure 4. Contact angle hysteresis of the probe liquids on the fluoropolymers: formamide (filled squares), ethylene glycol (filled diamonds), DMF (filled circles), $\alpha$-bromonaphthalene (filled pentagons), methylene iodide (hollow squares), bromoform (hollow diamonds), pyridine (hollow circles), and squalene (hollow pentagons).

interactions) between the probe liquids and the surface of PVDF. ${ }^{31,32}$ Zisman and co-workers interpreted a slight curvature in their plot for the copolymer of PTFE and PCTFE in terms of weak hydrogen-bonding interactions between the contacting liquids and the solid surface. ${ }^{33-35}$ We experimentally confirmed that polar protic liquids induced the upward curvature of the Zisman plot at the highest surface tension with water as the hydrogen-bonding probe liquid. On the other hand, ETFE, having weakly acidic hydrogens, exhibited no indication of a specific intermolecular interaction with water and showed an approximately linear Zisman plot for the polar protic liquids. As a whole, the results demonstrate that PVDF, which possesses the strongest surface dipoles and the most acidic hydrogens, undergoes the strongest non-dispersive interactions with probe liquids among all three partially fluorinated polymers.

Hysteresis of contact angles. Contact angle hysteresis is defined as the difference between the advancing contact angle and the receding contact angle $\left(\Delta \theta=\theta_{\mathrm{a}}-\theta_{\mathrm{r}}\right)$. Advancing and receding contact angles are known to probe, respectively, for the low-energy (or apolar) portion and the high-energy (or polar) portion of a surface. ${ }^{36,37}$ The main causes of hysteresis include absorption of moisture, functional groups in the interfacial region, and surface heterogeneity (such as surface contamination, reorientation, and roughness). ${ }^{38,39}$

Figure 4 shows a relatively small contact angle hysteresis ( $c a$. $10^{\circ}$ ) for all of the fluoropolymers except PVDF, which exhibits a large hysteresis $\left(c a \cdot 40^{\circ}\right)$. These results can be interpreted to indicate the operation of complex polar forces on the PVDF surface during contact angle measurements. In particular, the large hysteresis observed for polar protic liquids (such as formaldehyde and ethylene glycol) might suggest that the PVDF surface was inhomogeneous with respect to dispersive and/or complex polar forces, such as dipole-dipole interactions and/or acid-base interactions (including hydrogen bonding).

The vertical roughness of the polymer films measured by AFM topographical analysis is summarized in Table 1. Interestingly, ECTFE with the smallest hysteresis exhibited the largest 
Table 1. Lifshitz-van der Waals and acid-base components of fluoropolymers from the contact angles and surface tension data for the methylene iodide-water-glycerol system

\begin{tabular}{lccccc}
\hline Polymer & $\gamma_{\mathrm{S}}{ }^{\mathrm{LW}}$ & $\gamma_{\mathrm{S}}^{\mathrm{AB}}$ & $\gamma_{\mathrm{S}}{ }^{+}$ & $\gamma_{\mathrm{S}}{ }^{-}$ & $\mathrm{R}_{\mathrm{q}}{ }^{b}(\mathrm{~nm})$ \\
\hline FEP & $14.96\left(15.24^{\mathrm{a}}\right)$ & $0.18\left(0.17^{\mathrm{a}}\right)$ & $0.09\left(0.08^{a}\right)$ & $0.09\left(0.09^{a}\right)$ & 10.5 \\
PFA & $14.96\left(15.48^{\mathrm{a}}\right)$ & $0.47\left(0.42^{\mathrm{a}}\right)$ & $0.19\left(0.15^{a}\right)$ & $0.29\left(0.30^{a}\right)$ & 17.0 \\
ETFE & $17.48\left(16.51^{\mathrm{a}}\right)$ & $0.54\left(0.71^{\mathrm{a}}\right)$ & $0.08\left(0.13^{a}\right)$ & $0.91\left(0.96^{a}\right)$ & 11.1 \\
ECTFE & $29.73\left(28.90^{\mathrm{a}}\right)$ & $0.47\left(0.56^{\mathrm{a}}\right)$ & $0.14\left(0.19^{a}\right)$ & $0.39\left(0.41^{a}\right)$ & 29.1 \\
PVDF & $28.58\left(24.39^{\mathrm{a}}\right)$ & $1.76\left(3.75^{\mathrm{a}}\right)$ & $0.07\left(0.30^{a}\right)$ & $11.1\left(11.7^{a}\right)$ & 25.9 \\
\hline
\end{tabular}

All units in surface tension components for polymers in $\mathrm{mJ} \mathrm{m}^{-2}$. The values in Table 1 were taken from the previous paper. ${ }^{19}$ The surface tension components were calculated from the advancing contact angles based on the reference values for water: $\gamma_{\mathrm{L}}{ }^{+}={\gamma_{\mathrm{L}}}^{-}=25.5 .^{56}{ }^{a}$ Calculated using the nonzero value of $\gamma_{\mathrm{L}}{ }^{+}=$ 0.72 for methylene iodide. ${ }^{51}$ Root-mean-square (rms) surface roughness data measured by AFM.

surface roughness among the partially fluorinated polymers, which was much less than the threshold value $(\leq 50 \sim 100 \mathrm{~nm})$ that is usually considered to influence the contact angle hysteresis. ${ }^{40}$ Furthermore, analysis by XPS analysis showed no evidence for severe surface contamination and/or reconstruction (or reorientation). ${ }^{19}$ The absorption of moisture is not considered here because fluoropolymers are well-known to exhibit a low absorptivity of humidity and are inert to most chemicals., ${ }^{5,22}$

Work of adhesion. We employed a modified Young-GoodGirifalco-Fowkes method (i.e., the VCG method) and the Young-Dupré equation to investigate further the non-ideal wettability observed on the partially fluorinated polymer surfaces. ${ }^{41}$ In this analysis, we used three different types of contacting liquids to estimate the Lifshitz-van der Waals (LW) and the acid-base (AB) forces: (1) polar protic (water, formamide, and ethylene glycol), (2) polar aprotic (bifunctional: nitrobenzene, DMSO, and DMF; monofunctional: bromoform and pyridine), and (3) virtually apolar ( $\alpha$-bromonaphthalene). In particular, we examine the non-dispersive interactions (including surface dipoles and hydrogen bonding) in terms of the trends in wettability and the acid-base interfacial energies.

To determine the acid-base components by the VCG method, it is convenient to use equation 1 along with a virtually apolar probe liquid and two polar probe liquids having discrete values of $\gamma_{\mathrm{L}}{ }^{+}$and $\gamma_{\mathrm{L}}^{-41,42}$ :

$$
\gamma_{\mathrm{L}}\left(1+\cos \theta_{\mathrm{a}}\right)=2 \sqrt{\gamma_{\mathrm{S}}^{\mathrm{LW}} \gamma_{\mathrm{L}}^{\mathrm{LW}}}+2 \sqrt{\gamma_{\mathrm{S}}^{+} \gamma_{\mathrm{L}}^{-}}+2 \sqrt{\gamma_{\mathrm{S}}^{-} \gamma_{\mathrm{L}}^{-}}
$$

where $\gamma^{\mathrm{LW}}$ is the Lifshitz-van der Waals component, $\gamma^{+}$is the electron-acceptor (acidic) surface tension, and $\gamma^{-}$is the electrondonor (basic) surface tension. Using equation 1 and methylene iodide, water, and glycerol as probe liquids, we determined the acid-base components of the fluorinated polymers from the contact angles. As shown in Table 1, the nonzero $\gamma_{\mathrm{L}}{ }^{+}$value of methylene iodide revealed an increasing trend in acid-base interactions, $\gamma_{\mathrm{S}}^{\mathrm{AB}}$, for the partially fluorinated polymers as follows: ECTFE $<$ ETFE $<<$ PVDF.

Lifshitz-van der Waals (LW) forces: Using well-known lierature values of $\gamma_{\mathrm{L}}{ }^{\mathrm{LW}}$ for the contacting liquids (see Table 2) (2,43 $^{40}$ and $\gamma_{\mathrm{S}}{ }^{\mathrm{LW}}$ of the fluorinated polymers calculated from the nonzero value of $\gamma_{\mathrm{L}}{ }^{+}=0.72 \mathrm{~mJ} \mathrm{~m}^{-2}$ for methylene iodide and two polar liquids $\left(\gamma_{\mathrm{L}}^{+}=25.5\right.$ and $3.92 \mathrm{~mJ} \mathrm{~m}^{-2}$ for water and glycerol, respectively), ${ }^{44}$ we estimated the Lifshitz-van der Waals force $\left(W_{\mathrm{SL}}{ }^{\mathrm{LW}}\right)$ according to equation 2 and plotted the values of $W_{\mathrm{SL}}{ }^{\mathrm{LW}}$
Table 2. Acid-base components of various liquids cited by van $\mathrm{Oss}^{42,43}$

\begin{tabular}{lllccl}
\hline \multicolumn{1}{c}{ Liquids } & $\gamma_{\mathrm{L}}{ }^{\mathrm{LW}}$ & $\gamma_{\mathrm{L}}{ }^{\mathrm{AB}}$ & $\gamma_{\mathrm{L}}{ }^{+}$ & $\gamma_{\mathrm{L}}{ }^{-}$ & $\gamma_{\mathrm{L}}{ }^{\text {tot }}$ \\
\hline Water & 21.8 & 51 & 25.5 & 25.5 & 72.8 \\
Glycerol & 34 & 30 & 3.92 & 57.4 & 64 \\
Formamide & 39 & 19 & 2.28 & 39.6 & 58 \\
Ethylene Glycol & 29 & 19 & 1.92 & 47.0 & 48 \\
DMF & $30.2^{b}$ & 6.1 & $-^{a}$ & ${ }^{-}$ & 36.3 \\
Nitrobenzene & $38.7^{b}$ & 5.1 & $-^{a}$ & ${ }^{a}$ & 43.8 \\
DMSO & 36 & 8 & 0.5 & 32 & 44 \\
a-Bromonaphthalene & 43.5 & 0.09 & 0.39 & 0.48 & 44.4 \\
Bromoform & 41.5 & 0 & 1.72 & 0 & 41.5 \\
Methylene Iodide & 50.8 & 0 & 0.72 & 0 & 50.8 \\
Pyridine & 38.0 & 0 & 0 & ${ }^{a}$ & 38.0 \\
1,4-Dioxane & 33.5 & 0 & 0 & ${ }^{a}$ & 33.5 \\
Squalene & 29.2 & 0 & 0 & 0 & 29.2
\end{tabular}

${ }^{a}$ The value of the surface components is unavailable in the literature. ${ }^{b} \mathrm{We}$ used the $\gamma_{\mathrm{L}}{ }^{\mathrm{d}}$ value of Fowkes instead of van Oss due to the unavailability of the latter. ${ }^{52}$

for the respective fluorinated surfaces in Figure 5.

$$
W_{\mathrm{SL}}^{\mathrm{LW}}=2 \sqrt{\gamma_{\mathrm{S}}^{\mathrm{LW}} \gamma_{\mathrm{L}}^{\mathrm{LW}}}
$$

We note that the calculation of $W_{\mathrm{SL}}{ }^{\mathrm{LW}}$ based on the literature values of $\gamma_{\mathrm{L}}{ }^{\mathrm{LW}}$ might lead to errors in calculating interfacial energies because $\gamma_{\mathrm{L}}{ }^{\mathrm{LW}}$ for the contacting liquids were obtained through their interactions with hydrocarbon surfaces rather than fluorocarbon surfaces. ${ }^{18}$

Figure 5 shows that the values of $W_{\mathrm{SL}}{ }^{\mathrm{LW}}$ increase in the following order, regardless of the nature of the contacting liquids: FEP $<$ PFA $<$ ETFE $<$ PVDF $<<$ ECTFE. Since hydrogen is smaller than fluorine, it is reasonable that partially fluorinated ETFE (more densely packed) gives higher values of $W_{\mathrm{SL}}{ }^{\mathrm{LW}}$ than the fully fluorinated polymers. By the same analogy, PVDF might exhibit a higher $W_{\mathrm{SL}}{ }^{\mathrm{LW}}$ due to its higher molecular packing density than ETFE. ${ }^{16,19}$ We, however, cannot offer similar rationalization(s) for the enhanced $W_{\mathrm{SL}}^{\mathrm{LW}}$ for ECTFE. ECTFE exhibited the highest values of $W_{\mathrm{SL}}{ }^{\mathrm{LW}}$ for all of the contacting liquids, in spite of its lowest packing density among partiallyfluorinated polymers. It is possible that the enhanced $W_{\mathrm{SL}} \mathrm{LW}$ for ECTFE arises from intercalation of the contacting probe 
(a)

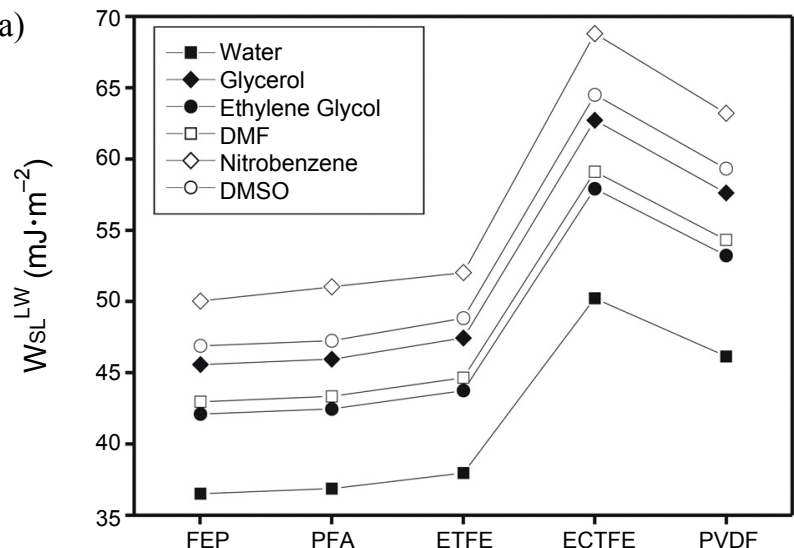

(b)

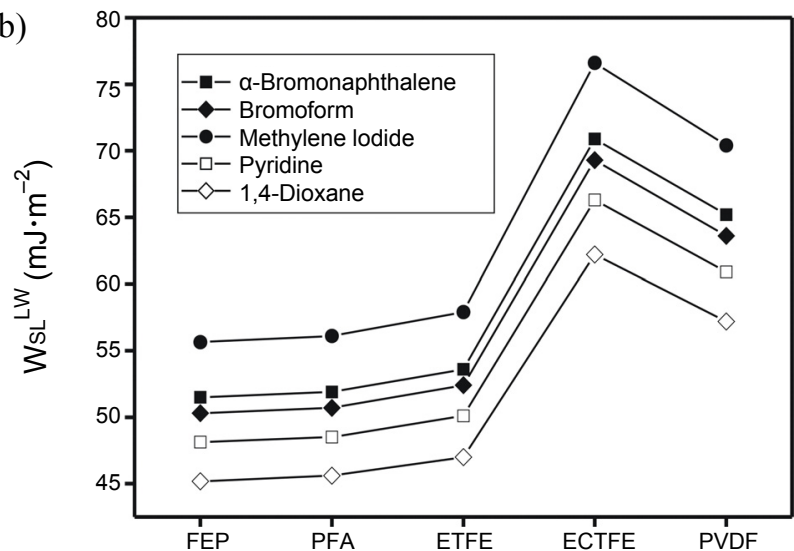

Figure 5. Nonpolar work of adhesion attributed to Lifshitz-van der Waals interactions between the probe liquids and the fluoropolymers. (a) Polar protic and bifunctional liquids: water (filled squares), glycerol (filled diamonds), ethylene glycol (filled circles), DMF (hollow squares), nitrobenzene (hollow diamonds), and DMSO (hollow circles). (b) Nonpolar and monofunctional liquids: $\alpha$-bromonaphthalene (filled squares), bromoform (filled diamonds), methylene iodide (filled circles), pyridine (hollow squares), and 1,4-dioxane (hollow diamonds).

liquids or because $\mathrm{Cl}$ is more polarizable than $\mathrm{F}$. Otherwise, the contribution of dipolar effects (including induction) on $W_{\mathrm{SL}}{ }^{\mathrm{LW}}$ might not be negligible due to the presence of chemical heterogeneity and multiple surface dipoles interacting with probe liquids. For Lifshitz-van der Waals (LW) interactions, by definition, comprise the dispersion, dipolar, and induction terms. ${ }^{41}$

Acid-Base (AB) interactions: Using an analogous strategy, we estimated the acid-base interactions $\left(W_{\mathrm{SL}}{ }^{\mathrm{AB}}\right)$ according to equation 3 and plotted the values of $W_{\mathrm{SL}}{ }^{\mathrm{AB}}$ for the respective fluorinated surfaces in Figure 6.

$$
W_{\mathrm{SL}}^{\mathrm{AB}}=\gamma_{\mathrm{L}}\left(1+\cos \theta_{\mathrm{a}}\right)-2 \sqrt{\gamma_{\mathrm{S}}^{\mathrm{LW}} \gamma_{\mathrm{L}}^{\mathrm{LW}}}
$$

As shown in Figure 6a and Table 3, polar protic and bifunctional polar aprotic liquids exhibited the same trends of $W_{\mathrm{SL}}{ }^{\mathrm{AB}}$ : ECTFE $<$ ETFE $<$ PVDF. Polar protic liquids exhibited a slightly higher $W_{\mathrm{SL}}{ }^{\mathrm{AB}}$ in comparison to those of bifunctional polar aprotic liquids, indicating the enhanced acid-base interaction including hydrogen-bonding of polar protic liquids. Notably, this enhancement was especially evident for water on the surface
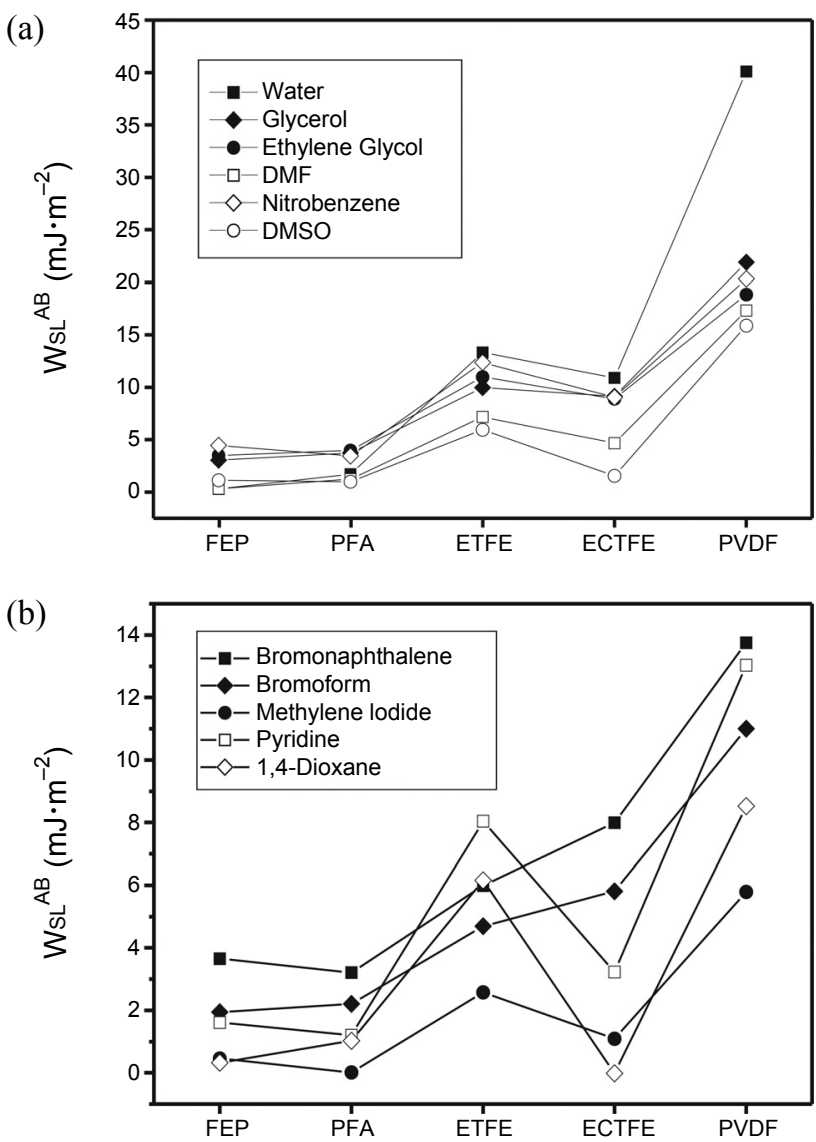

Figure 6. Polar work of adhesion attributed to acid-base interactions between the probe liquids and the fluoropolymers. (a) Polar protic and bifunctional liquids: water (filled squares), glycerol (filled diamonds), ethylene glycol (filled circles), DMF (hollow squares), nitrobenzene (hollow diamonds), and DMSO (hollow circles). (b) Nonpolar and monofunctional liquids: $\alpha$-bromonaphthalene (filled squares), bromoform (filled diamonds), methylene iodide (filled circles), pyridine (hollow squares), and 1,4-dioxane (hollow diamonds).

of PVDF, which possesses the most acidic hydrogens. If this enhancement were due to the surface acidity of PVDF, ethylene glycol (polar protic) should behave like water; pyridine and 1,4dioxane (monofunctional basic, Figure 6(b) should also behave like water. However, the results show an unusual enhancement only for water (Figure 6).

This discrepancy might arise from the characteristic property of water molecules to form hydrogen-bonded three dimensional clusters by self-association. ${ }^{45,46}$ While most other self-associating liquids usually form only dimers, water is known to construct a much more extended network that ranges from dimers to octamers. The three dimensional network of self-associated water molecules is maintained at non-polar hydrophobic surfaces, where non-polar dispersive interactions predominate between water and the surface. However, when so-called "stretched" water is in contact with polar hydrophilic surfaces, the Lewis acid/base sites on the surface disrupt the hydrogen bonds between water molecules, collapsing the extended network of water. ${ }^{47}$ The perturbation near the surface propagates into bulk water, leading to changes in water properties such as dipole moment and density. Since the disruption is caused by the 
Table 3. The calculated values of acid-base interaction energies of various liquids on partially fluorinated polymers

\begin{tabular}{lccccc}
\hline \multicolumn{1}{c}{ Liquids } & $W_{\mathrm{SL}}{ }^{\mathrm{AB}}(\mathrm{ETFE})$ & $W_{\mathrm{SL}}{ }^{\mathrm{AB}}(\mathrm{ECTFE})$ & $W_{\mathrm{SL}}{ }^{\mathrm{AB}}(\mathrm{PVDF})$ & $\gamma_{\mathrm{L}}{ }^{\mathrm{AB}}$ & Dipole Moment (D) \\
\hline Water & 13.30 & 10.87 & 40.1 & 51 & 1.84 \\
Glycerol & 9.94 & 9.11 & 21.88 & 30 & 3.0 \\
Formamide & 5.24 & 1.92 & 18.03 & 19 & 3.7 \\
Ethylene Glycol & 10.93 & 8.86 & 18.80 & 19 & 2.2 \\
DMF & 7.12 & 4.63 & 17.3 & 6.1 & 3.8 \\
Nitrobenzene & 12.34 & 8.99 & 20.3 & 5.1 & 4.2 \\
DMSO & 5.89 & 1.49 & 15.84 & 8 & 4.0 \\
Pyrrole & 5.02 & 5.88 & 17.00 & 4.8 & 1.8 \\
$\alpha$-Bromonaphthalene & 5.99 & 7.99 & 13.75 & 0.09 & 0 \\
Bromoform & 4.69 & 5.81 & 11.00 & 0 & 0 \\
Methylene Iodide & 2.57 & 1.09 & 5.79 & 0 & 0 \\
Pyridine & 8.04 & 3.23 & 13.03 & 0 & 0 \\
1,4-Dioxane & 6.16 & -0.02 & 8.52 & 0 & 0 \\
\hline
\end{tabular}

competition of the Lewis acid-base interaction between water and the surface with the self-association of water molecules, the strength and density of Lewis acid/base sites at the surface must be considered when interpreting wetting behavior. A previous AFM study investigated the surface forces acting on a series of partially alkylated silica surfaces with varying wettabilities. $^{48}$ The results revealed that attractive forces between water molecules are dominant at the surfaces on which the contact angle of water is higher than $62.4^{\circ}$, but repulsive forces become dominant at more wettable surfaces. ${ }^{47}$ This abrupt change in water association at a specific contact angle has been also observed on polymer surfaces. ${ }^{49}$ A plot of the surface free energies versus water contact angles on a series of polymer surfaces suggests that the dispersive component of surface energy suddenly levels out at $\theta=90^{\circ}$, which is higher than that observed with alkylated silica surfaces. ${ }^{47}$ According to the previous studies, we can reasonably argue that associative forces between water molecules predominate on $\operatorname{ETFE}\left(\theta=108^{\circ}\right)$ and $\operatorname{ECTFE}(\theta=$ $\left.99^{\circ}\right)$, but repulsive forces predominate on $\operatorname{PVDF}\left(\theta=80^{\circ}\right) .{ }^{19,47}$ In other words, water exists as self-associated clusters on the hydrophobic ETFE and ECTFE surfaces, but in a more disrupted state on the hydrophilic PVDF surfaces. The disrupted water molecules can have strong interactions with Lewis sites on the PVDF surface, which leads to the observed enhancement in wettability (Figure 6a).

Figure $6 \mathrm{~b}$ shows that the monobasic liquids, pyridine and 1,4-dioxane, exhibited stronger acid-base interactions with ETFE and PVDF than with ECTFE, showing the same trends of acid-base interaction energies observed with the polar protic and bifunctional liquids. Moreover, the monoacidic liquid, bromoform, ${ }^{50,51}$ exhibited stronger acid-base interactions with ECTFE than with ETFE, which correlates with our estimated order of surface acidity based on the inductive effects of surface dipoles: ECTFE $<$ ETFE $<<$ PVDF. Of the partially fluorinated polymers, ECTFE, with the least acidic hydrogens, exhibited the weakest $W_{\mathrm{SL}}{ }^{\mathrm{AB}}$ toward the monobasic liquids; in contrast, the $W_{\mathrm{SL}}{ }^{\mathrm{AB}}$ for ECTFE toward the monoacidic liquids (including virtually apolar $\alpha$-bromonaphthalene) was greater than that of ETFE (weakly acidic hydrogens), but weaker than that of PVDF (strongly acidic hydrogens). When taken as a whole, these data support a model in which there is a considerable contribution of acid-base interactions to the cumulative non-dispersive interactions.

The data in Table 1 indicate that the magnitude of the $\gamma_{\mathrm{s}}{ }^{-}$ monopole for the partially fluorinated polymers follows this order: ECTFE $<$ ETFE $<<<$ PVDF. Thus, strongly $\gamma_{\mathrm{S}}{ }^{-}$monopolar PVDF should interact with the $\gamma_{\mathrm{L}}{ }^{+}$monofunctional acids (bromoform and methylene iodide) more strongly than with the $\gamma_{\mathrm{L}}{ }^{-}$ monofunctional bases (pyridine and 1,4-dioxane). However, strongly $\gamma_{\mathrm{s}}^{-}$monopolar PVDF showed no preferential polar interactions toward the $\gamma_{\mathrm{L}}{ }^{+}$and $\gamma_{\mathrm{L}}{ }^{-}$monofunctional liquids. Furthermore, the least basic ECTFE should exhibit stronger acidbase interactions with monobasic liquids than does ETFE. Again, however, the experimental results are inconsistent with the prediction of the VCG method. Why the discrepancy? First, van Oss's expression of $W_{\mathrm{SL}}{ }^{\mathrm{AB}}$ offers only a limited expression of interfacial interactions (i.e., it overestimates the Lewis basicity, even for surfaces that are characteristically acidic ${ }^{52}$ ). Second, the strong dipole-dipole interactions acting across the interface ${ }^{19}$ might simply overwhelm the acid-base interactions. In any event, the experimental observations are consistent with a combination of polar effects (dipole-dipole and acid-base) influencing the wettability of the partially fluorinated surfaces.

Further evidence for the contribution of dipolar interactions to $W_{\mathrm{SL}}{ }^{\mathrm{AB}}$ is provided by the observation that virtually apolar $\alpha$ bromonaphthalene exhibited a higher $W_{\mathrm{SL}}{ }^{\mathrm{AB}}$ on ECTFE than ETFE, which is probably due to the anisotropic polarization of the probe liquid on the dipole-laden ECTFE surface, which leads to enhanced acid-base interactions for ECTFE. ${ }^{53,54}$ In addition, given the works of adhesion with the monobasic liquids, the data in Figure 6b strongly suggest that ECTFE (having the least acidic hydrogens among the partially fluorinated polymers) is relatively devoid of acid-base interactions; in contrast, ETFE and particularly PVDF (having the most acidic hydrogens among the partially fluorinated polymers) are relatively flush with acid-base interactions. Importantly, these observed trends in the works of adhesion mirror the estimated magnitude of the surface dipoles for these polymers. ${ }^{19}$ 


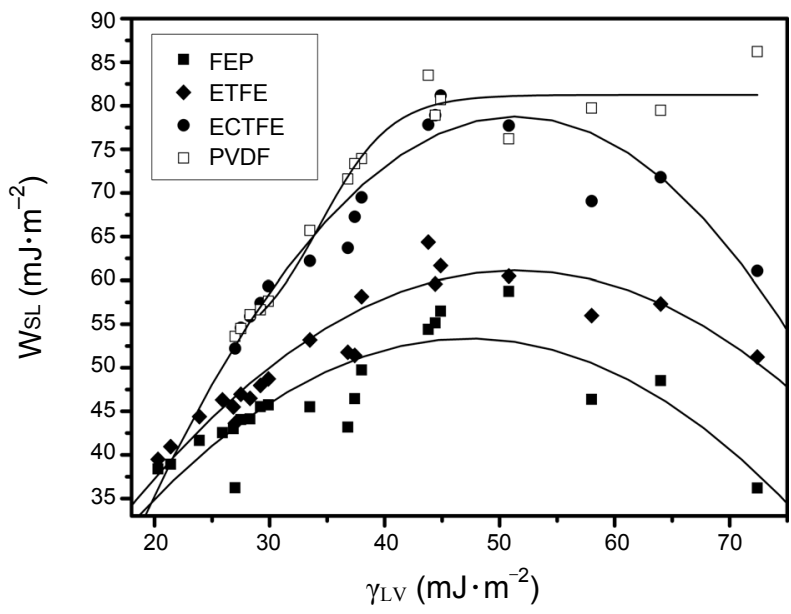

Figure 7. Effect of the liquid surface tension on the work of adhesion, $W_{\mathrm{SL}}$, for various fluoropolymers: FEP (filled squares), ETFE (filled diamonds), ECTFE (filled circles), and PVDF (hollow squares). The contacting liquids included water, formamide, glycerol, decanol, heptanol, DMF, $\alpha$-bromonaphthalene, nitrobenzene, methylene iodide, bromoform, pyridine, acetonitrile, 1,4-dioxane, pyrrole, heptane, octane, decane, tridecane, hexadecane, decalin, and squalene. For ECTFE and PVDF, heptanol, heptane, octane, decane, and tridecane were excluded because the surfaces were completely wet by these liquids (i.e., $\theta_{\mathrm{a}} \leq 10^{\circ}$ ).

Young-Dupré equation: We determined the total work of adhesion, $W_{\mathrm{SL}}$, from the measured contact angles and the liquid surface tensions according to the Young-Dupré equation: $W_{\mathrm{SL}}=$ $\gamma_{\mathrm{LV}}\left(1+\cos \theta_{a}\right){ }^{17,18}$ As shown in Figure 7, the total work of adhesion, $W_{\mathrm{SL}}$, on FEP, ETFE, and ECTFE exhibited a maximum value for the probe liquids having intermediate surface tensions (i.e., $\gamma_{\mathrm{LV}} \sim 50 \mathrm{~mJ} \mathrm{~m}^{-2}$ ), with lower values for lower and higher surface tensions. In contrast, $W_{\mathrm{SL}}$ for PVDF rose steadily with surface tension and then became constant; this behavior is consistent with a model in which strong acid-base interactions (including hydrogen bonding) occur between the polar protic liquids (especially water) and the PVDF surface. ${ }^{55}$ Such interactions between water and PVDF can disrupt the hydrogen-bonding network in water clusters, leading to remarkably strong acid-base interactions across the interface.

\section{Conclusions}

The wettability of fluoropolymer surfaces was evaluated using a combination of polar protic, polar aprotic (bifunctional and monofunctional), and virtually nonpolar contacting liquids. A Zisman plot of $\cos \theta_{a} v s . \gamma_{\mathrm{LV}}$ for partially fluorinated ETFE and ECTFE exhibited an approximate linearity for polar protic contacting liquids, while PVDF exhibited a slight upward curvature deviated from linearity, suggesting strong non-dispersive interactions between the PVDF surface and the polar protic liquids. The relative strengths of the acid-base interaction energies estimated for the partially fluorinated surfaces using the VCG method (i.e., $\gamma_{\mathrm{S}}{ }^{+}$and $\gamma_{\mathrm{S}}{ }^{-}$) were oftentimes inconsistent with the relative strengths of the acid-base interaction energies of the probe liquids (i.e., $\gamma_{\mathrm{L}}{ }^{+}$and $\gamma_{\mathrm{L}}{ }^{-}$), which was interpreted to indicate a considerable contribution of dipole-dipole interactions to the total non-dispersive interactions. Notably, PVDF with the most acidic hydrogens exhibited acid-base interactions that were surprisingly strong (especially for water). Apparently, the strong Lewis sites on the PVDF surface interrupt the hydrogen-bonding network of bulk water, leading to an unusually enhanced wettability by water of the PVDF surface.

Acknowledgments. We thank our colleague David Barriet for experimental assistance and helpful discussions and Dr. K. Y. Young (Materials Lab., Samsung Advanced Institute of Technology) for helpful discussions regarding the AFM studies. The work was supported by the Kyungwon University Research Fund in 2010, the National Research Foundation of Korea Grant (NRF-2010-C1AAA001-2010-0028958), the National Science Foundation (DMR-0906727), and the Robert A. Welch Foundation (E-1320).

\section{References}

1. Fluoropolymers; Hougham, G., Cassidy, P. E., Johns, K., Davidson, T., Eds.; Plenum: New York, 1999.

2. Kinloch, A. J. Adhesion and Adhesives; Chapman and Hall: New York, 1987.

3. Imbalazano, J. F. Chem. Eng. Prog. 1991, 87, 69-73.

4. Pittman, A. G. In Fluoropolymers; Wall, L. A., Ed.; Wiley: New York, 1971; Vol. 25, p 419.

5. Modern Fluoropolymers: High Performance Polymers for Diverse Applications; Scheirs, J., Ed.; Wiley: New York, 1997.

6. Bottino, A.; Capannelli, G.; Monticelli, O.; Piaggio, P. J. Membrane Sci. 2000, 166, 23-29.

7. Wang, P.; Zakeeruddin, S. M.; Moser, J. E.; Nazeeruddin, M. K.; Sekiguchi, T.; Graetzel, M. Nat. Mater. 2003, 2, 402-407.

8. Arora, P.; Zhang, Z. Chem. Rev. 2004, 104, 4419-4462.

9. Stephan, A. M.; Nahm, K. S. Polymer 2006, 47, 5952-5964.

10. Panero, S.; Ciuffa, F.; D'Epifano, A.; Scrosati, B. Electrochim. Acta 2003, 48, 2009-2014.

11. Mokrini, A.; Huneault, M. A. J. Power Sources 2006, 154, 51-58.

12. Chen, J.; Asano, M.; Maekawa, Y.; Yoshida, M. J. Membrane Sci. 2006, 277, 249-257.

13. Benz, M.; Euler, W. B.; Gregory, O. J. Macromolecules 2002, 35, 2682-2688.

14. Ueberschlag, P. Sensor Review 2001, 21, 118-126.

15. Cho, K.-Y.; Eom, J.-Y.; Jung, H.-Y.; Choi, N.-S.; Lee, Y. M.; Park, J.-K.; Choi, J.-H.; Park, K.-W.; Sung, Y.-E. Electrochim. Acta 2004, 50, 583-588.

16. Ellison, A. H.; Zisman, W. A. J. Phys. Chem. 1954, 58, 260-5.

17. Colorado, R., Jr.; Lee, T. R. J. Phys. Org. Chem. 2000, 13, 796-807.

18. Colorado, R., Jr.; Lee, T. R. Langmuir 2003, 19, 3288-3296.

19. Lee, S.; Park, J.-S.; Lee, T. R. Langmuir 2008, 24, 4817-4826.

20. Lee, S.; Knaebel, K. S. J. Appl. Polym. Sci. 1997, 64, 455-476.

21. Benz, M.; Euler, W. B.; Gregory, O. J. Langmuir 2001, 17, 239243.

22. Encyclopedia of Polymer Science and Engineering, 2nd ed.; Mark, H. F., Bikales, N. M., Overberger, C. G., Menges, G., Eds.; Wiley: New York, 1986; Vol. 16.

23. Dikshit, A. K.; Nandi, A. K. Macromolecules 2000, 33, 2616-2625.

24. Gao, Q.; Scheinbeim, J. I. Macromolecules 2000, 33, 7564-7572.

25. Holman, R. W.; Kavarnos, G. J. Polymer 1996, 37, 1697-1701.

26. Lee, S. Ph.D. Thesis, Ohio State University, 1995.

27. Radice, S.; Del Fanti, N.; Castiglioni, C.; Del Zoppo, M.; Zerbi, G. Macromolecules 1994, 27, 2194-9.

28. Radice, S.; Del Fanti, N.; Zerbi, G. Polymer 1997, 38, 2753-2758.

29. Zisman, W. A. Adv. Chem. Ser. 1964, 43, 1-51.

30. Chen, F.; Chang, W. V. Langmuir 1991, 7, 2401-4.

31. Jie-Rong, C.; Wakida, T. J. Appl. Polym. Sci. 1997, 63, 1733-1739.

32. Bernett, M. K.; Zisman, W. A. J. Phys. Chem. 1960, 64, 1292-4.

33. Baier, R. E.; Zisman, W. A. Macromolecules 1970, 3, 462-8. 
34. Fox, H. W.; Zisman, W. A. J. Colloid Sci. 1952, 7, 109-21.

35. Baier, R. E.; Zisman, W. A. Macromolecules 1970, 3, 70-9.

36. Morra, M.; Occhiello, E.; Garbassi, F. Langmuir 1989, 5, 872-876.

37. Tretinnikov, O. N. J. Colloid Interf. Sci. 2000, 229, 644-647.

38. Extrand, C. W. J. Colloid Interf. Sci. 2002, 248, 136-142.

39. Shon, Y.-S.; Lee, S.; Colorado, R., Jr.; Perry, S. S.; Lee, T. R. J. Am. Chem. Soc. 2000, 122, 7556-7563.

40. De Gennes, P. G. Rev. Mod. Phys. 1985, 57, 827-63.

41. van Oss, C. J.; Chaudhury, M. K.; Good, R. J. Chem. Rev. 1988, 88 , 927-41.

42. van Oss, C. J.; Giese, R. F., Jr.; Good, R. J. Langmuir 1990, 6, 171113.

43. Wu, W.; Giese, R. F., Jr.; van Oss, C. J. Langmuir 1995, 11, 379-82.

44. Janczuk, B.; Bialopiotrowicz, T.; Zdziennicka, A. J. Colloid Interf. Sci. 1999, 211, 96-103.

45. Gregory, J. K.; Clary, D. C.; Liu, K.; Brown, M. G.; Saykally, R. J. Science 1997, 275, 814-817.

46. Silvestrelli, P. L.; Parrinello, M. Phys. Rev. Lett. 1999, 82, 3308.
47. Vogler, E. A. Adv. Colloid Interface Sci. 1998, 74, 69-117.

48. Yoon, R.-H.; Flinn, D. H.; Rabinovich, Y. I. J. Colloid Interf. Sci. 1997, 185, 363-370.

49. Schakenraad, J. M.; Busscher, H. J.; Wildevuur, C. R. H.; Arends, J. J. Biomed. Mater. Res. 1986, 20, 773-784.

50. Janczuk, B.; Kerkeb, M. L.; Bialopiotrowicz, T.; GonzalezCaballero, F. J. Colloid Interf. Sci. 1992, 151, 333-42.

51. Janczuk, B.; Wojcik, W.; Zdziennicka, A. J. Colloid Interf. Sci. 1993, 157, 384-93.

52. Fowkes, F. M.; Riddle, F. L., Jr.; Pastore, W. E.; Weber, A. A. Colloid. Surface 1990, 43, 367-87.

53. Metrangolo, P.; Resnati, G. Chem. Eur. J. 2001, 7, 2511-2519.

54. Fourmigue, M. Curr. Opin. Solid State Mater. Sci. 2009, 13, 36-45.

55. Zisman, W. A. In Proceedings of the Symposium on Adhesion and Cohesion; Weiss, P., Ed.; Elsevier: Amsterdam, 1962; pp 176-208.

56. van Oss, C. J.; Good, R. J.; Chaudhury, M. K. Langmuir 1988, 4, 884-91. 